\title{
Adaptive control with convex saturation constraints
}

\author{
Jin Yan, Davi Antônio dos Santos, Dennis S. Bernstein \\ Department of Aerospace Engineering, The University of Michigan, Ann Arbor, MI, USA \\ E-mail:yanjin@umich.edu
}

\begin{abstract}
This study applies retrospective cost adaptive control to command following in the presence of multi-variable convex input saturation constraints. To account for the saturation constraint, the authors use convex optimisation to minimise the quadratic retrospective cost function. The use of convex optimisation bounds the magnitude of the retrospectively optimised input and thereby influences the controller update to satisfy the control bounds. This technique is applied to a multi-rotor helicopter with constraints on the total thrust magnitude and inclination of the rotor plane.
\end{abstract}

\section{Introduction}

All real-world control systems must operate subject to constraints on the allowable control inputs. These constraints typically have the form of a saturation input non-linearity [1]. The effects of saturation are addressed through antiwindup strategies [2-7]. Within the context of modern multi-variable control, techniques for dealing with saturation are presented in [8-12]. Saturation within the context of adaptive control is addressed in [13-16].

In the case of multiple control inputs, it is usually the case that individual control inputs are subject to independent saturation [17]. However, in many applications, a saturation constraint may constrain multiple control inputs. This is the case, for example, if the control inputs are produced by common hardware, such as a single power supply, amplifier or actuator.

In the present paper, we consider an adaptive control for problems, in which multiple control inputs may be subject to dependent saturation constraints. In particular, we are motivated by the problem of safely controlling the trajectory of a multi-rotor helicopter by constraining the total thrust magnitude and inclination in order to restrict the vehicle acceleration.

To address this problem, we revisit the problem of retrospective cost adaptive control (RCAC) under constraints [15]. RCAC can be used for adaptive command following and disturbance rejection for possibly non-minimumphase systems under minimal modelling information [18-21]. Unlike [17], the present paper uses convex optimisation to perform the retrospective input optimisation [22]. The use of convex optimisation bounds the magnitude of the retrospectively optimised input and thereby influences the controller update to satisfy the control bounds. We demonstrate this technique on illustrative numerical examples involving single and multiple inputs. We then apply this approach to trajectory control for a multi-rotor helicopter. We use the convex programming code [23] for the numerical optimisation. A related technique was used within the context of RCAC in [24] to address the problem of unknown non-minimum-phase zeros.

The contents of the paper are as follows. In Section 2, we describe the command-following problem with input saturation non-linearities. In Section 3, we summarise the RCAC algorithm. Numerical simulation results are presented in Section 4, and conclusions are given in Section 5.

\section{Problem formulation}

Consider the multiple-input multiple-output (MIMO) discrete-time Hammerstein system

$$
x(k+1)=A x(k)+B \operatorname{Sat}(u(k))+D_{1} w(k)
$$

where, for all $k \geq 0, x(k) \in \mathbb{R}^{n}, y(k) \in \mathbb{R}^{l_{y}}, z(k) \in \mathbb{R}^{l_{z}}$, $w(k) \in \mathbb{R}^{l_{w}}$ and $u(k) \in \mathbb{R}^{l_{u}}$. The signal $u(k)$ is the commanded control input, and $w(k)$ is exogenous signal. However, because of saturation, the actual control input is given by $v(k)=\operatorname{sat}(u(k))$, where the saturation input non-linearity is sat: $\mathbb{R}^{l_{u}} \rightarrow \mathcal{U}$, and $\mathcal{U} \subseteq \mathbb{R}^{l_{u}}$ is the convex control constraint set. We assume that the function 'Sat' is onto, that is, $\operatorname{sat}\left(\mathbb{R}^{l_{u}}\right)=\mathcal{U}$. In particular, if $\mathcal{U}$ is rectangular, then

$$
\operatorname{Sat}(u)=\left[\begin{array}{c}
\operatorname{sat}_{a_{1}, b_{1}}\left(u_{1}\right) \\
\vdots \\
\operatorname{sat}_{a_{l_{u}}, b_{l_{u}}}\left(u_{l_{u}}\right)
\end{array}\right]
$$

where $u=\left[\begin{array}{lll}u_{1} & \cdots & u_{l_{u}}\end{array}\right]^{\mathrm{T}} \in \mathcal{U}=\left[a_{1}, b_{1}\right] \times \cdots \times\left[a_{l_{u}}, b_{l_{u}}\right]$ and sat $: \mathbb{R} \rightarrow[a, b]$ is defined as

$$
\operatorname{sat}_{a, b}(u)= \begin{cases}a, & \text { if } u<a \\ u, & \text { if } a \leq u \leq b \\ b, & \text { if } u>b\end{cases}
$$

We consider the Hammerstein command-following and disturbance rejection problem with the performance variable

$$
z(k)=E_{1} x(k)+E_{0} w(k)
$$


and we assume that measurements of $z(k)$ are available for feedback; however, measurements of sat $(u(k))$ are not available. The goal is to develop an adaptive output feedback controller that minimises the performance error $z(k)$ with minimal modelling information about the plant dynamics, exogenous signal $w$ and input saturation non-linearity sat. Note that $w$ can represent either a command signal to be followed, an external disturbance to be rejected or both. For example, if $D_{1}=0$ and $E_{0} \neq 0$, then the objective is to have the output $E_{1} x$ follow the command signal $-E_{0} w$. On the other hand, if $D_{1} \neq 0$ and $E_{0}=0$, then the objective is to reject the disturbance $w$ from the performance variable $E_{1} x$. The combined command-following and disturbancerejection problem is considered when $D_{1}=\left[\begin{array}{ll}D_{11} & 0\end{array}\right]$, $E_{0}=\left[\begin{array}{ll}0 & E_{02}\end{array}\right]$ and $w(k)=\left[\begin{array}{lll}w_{1}^{\mathrm{T}}(k) & w_{2}^{\mathrm{T}}(k)\end{array}\right]^{\mathrm{T}}$, where the objective is to have $E_{1} x$ follow $-E_{0} w_{2}$ while rejecting the disturbance $w_{1}$. Finally, if $D_{1}$ and $E_{0}$ are zero matrices, then the objective is output stabilisation, that is, convergence of $z$ to zero.

\section{Retrospective cost adaptive control}

In this section, we describe the constrained retrospective cost optimisation algorithm.

\subsection{ARMAX modelling}

Consider the autoregressive-moving-average model with exogenous inputs (ARMAX) representation of (1)-(4) given by

$$
\begin{aligned}
z(k)= & \sum_{i=1}^{n}-\alpha_{i} z(k-i)+\sum_{i=d}^{n} \beta_{i} \operatorname{sat}(u(k-i)) \\
& +\sum_{i=0}^{n} \gamma_{i} w(k-i)
\end{aligned}
$$

where $\alpha_{1}, \ldots, \alpha_{n} \in \mathbb{R}, \beta_{1}, \ldots, \beta_{n} \in \mathbb{R}^{l_{z} \times l_{u}}, \gamma_{0}, \ldots, \gamma_{n} \in \mathbb{R}^{l_{z} \times l_{w}}$ and $d$ is the relative degree. Next, let $v(k) \triangleq \operatorname{Sat}(u(k))$, and define the transfer function

$$
G_{z v}(\mathbf{q}) \triangleq E_{1}(\mathbf{q} I-A)^{-1} B=\sum_{i=d}^{\infty} \mathbf{q}^{-i} H_{i}=H_{d} \frac{\alpha(\mathbf{q})}{\beta(\mathbf{q})}
$$

where $\mathbf{q}$ is forward shift operator and, for each positive integer $i$, the Markov parameter $H_{i}$ of $G_{z v}$ is defined by

$$
H_{i} \triangleq E_{1} A^{i-1} B \in \mathbb{R}^{l_{z} \times l_{u}}
$$

Note that, if $d=1$, then $H_{1}=\beta_{1}$, whereas, if $d \geq 2$, then

$$
\beta_{1}=\cdots=\beta_{d-1}=H_{1}=\cdots=H_{d-1}=0
$$

and $H_{d}=\beta_{d}$. The polynomials $\alpha(\mathbf{q})$ and $\beta(\mathbf{q})$ have the form

$$
\begin{aligned}
& \alpha(\mathbf{q})=\mathbf{q}^{n-1}+\alpha_{1} \mathbf{q}^{n-1}+\cdots+\alpha_{n-1} \mathbf{q}+\alpha_{n} \\
& \beta(\mathbf{q})=\mathbf{q}^{n-d}+\beta_{d+1} \mathbf{q}^{n-d-1}+\cdots+\beta_{n-1} \mathbf{q}+\beta_{n}
\end{aligned}
$$

Next, define the extended performance $Z(k) \in \mathbb{R}^{p_{z}}$ and extended plant input $V(k) \in \mathbb{R}^{q_{c} l_{u}}$ by

$$
\begin{aligned}
Z(k) & \triangleq\left[\begin{array}{c}
z(k) \\
\vdots \\
z(k-p+1)
\end{array}\right], \quad V(k) \triangleq\left[\begin{array}{c}
v(k-1) \\
\vdots \\
v\left(k-q_{c}\right)
\end{array}\right] \\
& =\left[\begin{array}{c}
\operatorname{sat}(u(k-1)) \\
\vdots \\
\operatorname{sat}\left(u\left(k-q_{c}\right)\right)
\end{array}\right]
\end{aligned}
$$

where the data window size $p$ is a positive integer, and $q_{c} \triangleq$ $n+p-1$. Therefore (11) can be expressed as

$$
Z(k)=W_{z w} \phi_{z w}(k)+B_{f} V(k)
$$

where (see (13))

$$
B_{f} \triangleq\left[\begin{array}{cccccc}
\beta_{1} & \cdots & \beta_{n} & 0_{l_{z} \times l_{u}} & \cdots & 0_{l_{z} \times l_{u}} \\
0_{l_{z} \times l_{u}} & \ddots & & \ddots & \ddots & \vdots \\
\vdots & \ddots & \ddots & & \ddots & 0_{l_{z} \times l_{u}} \\
0_{l_{z} \times l_{u}} & \cdots & 0_{l_{z} \times l_{u}} & \beta_{1} & \cdots & \beta_{n}
\end{array}\right] \in \mathbb{R}^{p l_{z} \times q_{c} l_{u}}
$$

and

$$
\phi_{z w}(k) \triangleq\left[\begin{array}{c}
z(k-1) \\
\vdots \\
z(k-p-n+1) \\
w(k) \\
\vdots \\
w(k-p-n+1)
\end{array}\right] \in \mathbb{R}^{q_{c} l_{z}+\left(q_{c}+1\right) l_{w}}
$$

Note that $W_{z w}$ includes modelling information about the poles of $G_{z v}$ and the exogenous signals, whereas $B_{f}$ includes modelling information about the zeros of $G_{z v}$.

For the open-loop system (5), we make the following assumptions:

(A.1) The relative degree $d$ is known.

(A.2) The first non-zero Markov parameter $H_{d}$ is known.

(A.3) There exists an integer $\bar{n}$ such that $n<\bar{n}$ and $\bar{n}$ is known.

(A.4) If $\zeta \in \mathbb{C},|\zeta|>1$, and $\beta(\zeta)=0$, then the spectral radius of $A$ is less than 1 .

(A.5) The performance variable $z(k)$ is measured and available for feedback.

(A.6) Each component of the function Sat is monotonically non-decreasing in each component in $u$ with the remaining components of $u$ fixed.

$$
W_{z w} \triangleq\left[\begin{array}{cccccccccccc}
-\alpha_{1} I_{l_{z}} & \cdots & -\alpha_{n} I_{l_{z}} & 0_{l_{z} \times l_{z}} & \cdots & 0_{l_{z} \times l_{z}} & \gamma_{0} & \cdots & \gamma_{n} & 0_{l_{z} \times l_{w}} & \cdots & 0_{l_{z} \times l_{w}} \\
0_{l_{z} \times l_{z}} & \ddots & & \ddots & \ddots & \vdots & 0_{l_{z} \times l_{w}} & \ddots & & \ddots & \ddots & \vdots \\
\vdots & \ddots & \ddots & & \ddots & 0_{l_{z} \times l_{z}} & \vdots & \ddots & \ddots & & \ddots & 0_{l_{z} \times l_{w}} \\
0_{l_{z} \times l_{z}} & \cdots & 0_{l_{z} \times l_{z}} & -\alpha_{1} I_{l_{z}} & \cdots & -\alpha_{n} I_{l_{z}} & 0_{l_{z} \times l_{w}} & \cdots & 0_{l_{z} \times l_{w}} & \gamma_{0} & \cdots & \gamma_{n}
\end{array}\right] \in \mathbb{R}^{p l_{z} \times\left[q_{c} l_{z}+\left(q_{c}+1\right) l_{w}\right]}
$$


(A.7) The exogenous signal $w(k)$ is generated by

$$
\begin{gathered}
x_{w}(k+1)=A_{w} x_{w}(k) \\
w(k)=C_{w} x_{w}(k)
\end{gathered}
$$

where $x_{w} \in \mathbb{R}^{l_{w}}$ and all of the eigenvalues of $A_{w}$ are on the unit circle and do not coincide with the transmission zeros of $G_{z v}$.

(A.8) There exists an integer $\bar{n}_{w}$ such that $n_{w}<\bar{n}_{w}$ and $\bar{n}_{w}$ is known.

(A.9) The exogenous signal $w(k)$ is not measured.

(A.10) $\alpha(\mathbf{q}), \beta(\mathbf{q}), n$ and $x(0)$ are unknown.

The Assumption 3.1 is motivated by Yan and Bernstein [25], where it is shown that monotonicity of the input non-linearity preserves the signs of the Markov parameters of the linearised system.

\subsection{Controller construction}

The commanded control $u(k)$ is given by the exactly proper time-series controller

$$
u(k)=\sum_{i=1}^{n_{\mathrm{c}}} M_{i}(k) u(k-i)+\sum_{j=0}^{n_{\mathrm{c}}} N_{j}(k) z(k-j)
$$

where, for all $i=1, \ldots, n_{\mathrm{c}}, M_{i}(k) \in \mathbb{R}^{l_{u} \times l_{u}}$, and, for all $j=0, \ldots, n_{\mathrm{c}}, N_{j}(k) \in \mathbb{R}^{l_{u} \times l_{z}}$. We express (18) as

$$
u(k)=\theta(k) \phi(k-1)
$$

where

$$
\begin{aligned}
\theta(k) & \triangleq\left[\begin{array}{llllll}
M_{1}(k) & \cdots & M_{n_{\mathrm{c}}}(k) & N_{0}(k) & \cdots & N_{n_{\mathrm{c}}}(k)
\end{array}\right] \\
& \in \mathbb{R}^{l_{u} \times\left(n_{\mathrm{c}} l_{u}+\left(n_{\mathrm{c}}+1\right) l_{z}\right)}
\end{aligned}
$$

and

$$
\phi(k-1) \triangleq\left[\begin{array}{c}
u(k-1) \\
\vdots \\
u\left(k-n_{\mathrm{c}}\right) \\
z(k) \\
\vdots \\
z\left(k-n_{\mathrm{c}}\right)
\end{array}\right] \in \mathbb{R}^{n_{\mathrm{c}} l_{u}+\left(n_{\mathrm{c}}+1\right) l_{z}}
$$

\subsection{Retrospective performance}

Define the retrospective performance $\hat{Z}(k) \in \mathbb{R}^{p l_{z}}$ by

$$
\hat{Z}(k) \triangleq W_{z w} \phi_{z w}(k)+B_{f} V(k)+\bar{B}_{f}[\hat{U}(k)-U(k)]
$$

where (see (23))

is the retrospective input matrix with the model information of $G_{z v}$. Specifically, $\bar{H}_{1}, \ldots, \bar{H}_{m}$ in (23) are estimates of the Markov parameters of $G_{z v}$, where $m \in \mathbb{Z}^{+}$. Next, define the extended commanded control $U(k) \in \mathbb{R}^{q_{c} l_{u}}$ and the retrospectively optimised extended control vector $\hat{U}(k) \in \mathbb{R}^{q_{c} l_{u}}$ by

$$
U(k) \triangleq\left[\begin{array}{c}
u(k-1) \\
\vdots \\
u\left(k-q_{c}\right)
\end{array}\right] \text { and } \hat{U}(k) \triangleq\left[\begin{array}{c}
\hat{u}_{k}(k-1) \\
\vdots \\
\hat{u}_{k}\left(k-q_{c}\right)
\end{array}\right]
$$

where $\hat{u}_{k}(k-i) \in \mathbb{R}^{l_{u}}$ is a recomputed control. Subtracting (12) from (22) yields

$$
\hat{Z}(k)=Z(k)+\bar{B}_{f}[\hat{U}(k)-U(k)]
$$

Note that the retrospective performance $\hat{Z}(k)$ does not depend on $W_{z w}$ or the exogenous signal $w$. For disturbance rejection, we do not assume that the disturbance is known; for command-following, the command-following error is needed but the command $w$ need not be separately measured. The model information matrix $\bar{B}_{f}$ is discussed in Section 3.5.

\subsection{Retrospective cost and RLS controller update law}

3.4.1 Retrospective cost: We define the retrospective cost function

$$
J(\hat{U}(k), k) \triangleq \hat{Z}^{\mathrm{T}}(k) R(k) \hat{Z}(k)+\eta(k) \hat{U}(k)^{\mathrm{T}} \hat{U}(k)
$$

where, for all $k>0, \eta(k) \geq 0$ is a scalar and $R(k) \in \mathbb{R}^{p l_{z} \times p l_{z}}$ is a positive-definite performance weighting. The goal is to determine retrospectively optimised controls $\hat{U}(k)$ that would have provided better performance than the controls $U(k)$ that were applied to the plant. The retrospectively optimised controls $\hat{U}(k)$ are subsequently used to update the controller. Using (25), (26) can be rewritten as

$$
J(\hat{U}(k), k)=\hat{U}(k)^{\mathrm{T}} \mathcal{A}(k) \hat{U}(k)+\mathcal{B}(k) \hat{U}(k)+\mathcal{C}(k)
$$

where

$$
\begin{aligned}
\mathcal{A}(k) \triangleq & \bar{B}_{f}^{\mathrm{T}} R(k) \bar{B}_{f}+\eta(k) I_{q_{c} l_{u}} \\
\mathcal{B}(k) \triangleq & 2 \bar{B}_{f}^{\mathrm{T}} R(k)\left[Z(k)-\bar{B}_{f} U(k)\right] \\
\mathcal{C}(k) \triangleq & Z^{\mathrm{T}}(k) R(k) Z(k)-2 Z^{\mathrm{T}}(k) R(k) \bar{B}_{f} U(k) \\
& +U(k)^{\mathrm{T}} \bar{B}_{f}^{\mathrm{T}} R(k) \bar{B}_{f} U(k)
\end{aligned}
$$

Note that if either $\bar{B}_{f}$ has full rank or $\eta(k)>0$, then $\mathcal{A}(k)$ is positive definite.

Next, we consider the problem of minimising (26) subject to

$$
\hat{U}(k) \in \mathcal{U} \times \cdots \times \mathcal{U}
$$

The following result follows from the Weierstrass theorem.

Lemma 3.1: If $\mathcal{U}$ is compact, then (26) has at least one minimiser. If, in addition, $\mathcal{U}$ is convex, then (26) has a unique

$$
\bar{B}_{f} \triangleq\left[\begin{array}{cccccccccc}
0_{l_{z} \times(d-1) l_{u}} & \bar{H}_{d} & \cdots & \bar{H}_{m} & 0_{l_{z} \times l_{u}} & \cdots & 0_{l_{z} \times l_{u}} & 0_{l_{z} \times l_{u}} & \cdots & 0_{l_{z} \times l_{u}} \\
0_{l_{z} \times(d-1) l_{u}} & 0_{l_{z} \times l_{u}} & \ddots & & \ddots & \ddots & & \ddots & \ddots & \vdots \\
\vdots & \vdots & \ddots & \ddots & & \ddots & \ddots & & \ddots & \vdots \\
0_{l_{z} \times(d-1) l_{u}} & 0_{l_{z} \times l_{u}} & \cdots & 0_{l_{z} \times l_{u}} & \bar{H}_{d} & \cdots & \bar{H}_{m} & 0_{l_{z} \times l_{u}} & \cdots & 0_{l_{z} \times l_{u}}
\end{array}\right] \in \mathbb{R}^{p l_{z} \times q_{c} l_{u}}
$$


minimiser. In particular, if $\mathcal{U}=\mathbb{R}^{l_{u}}$, then the unique global minimiser of $J(\hat{U}(k), k)$ is

$$
\hat{U}(k)=-\frac{1}{2} \mathcal{A}^{-1}(k) \mathcal{B}(k)
$$

3.4.2 Cumulative cost and RLS update: Define the cumulative cost function

$$
\begin{aligned}
J_{\text {cum }}(\theta, k) \triangleq & \sum_{i=d+1}^{k} \lambda^{k-i}\left\|\phi^{\mathrm{T}}(i-d-1) \theta(i-1)-\hat{u}_{k}(i-d)\right\|^{2} \\
& +\lambda^{k}[\theta(k)-\theta(0)]^{\mathrm{T}} P_{0}^{-1}[\theta(k)-\theta(0)]
\end{aligned}
$$

where $\|\cdot\|$ is the Euclidean norm, $P_{0} \in$ $\mathbb{R}^{l_{u}\left[n_{\mathrm{c}} l_{u}+\left(n_{\mathrm{c}}+1\right) l_{z}\right] \times\left[n_{\mathrm{c}} l_{u}+\left(n_{\mathrm{c}}+1\right) l_{z}\right]}$ is positive definite, and $\lambda \in(0,1]$ is the forgetting factor. The next result follows from standard recursive least-squares (RLS) theory [26, 27].

Lemma 3.2: For each $k \geq d$, the unique global minimiser of the cumulative retrospective cost function (30) is given by

$$
\theta(k)=\theta(k-1)+\frac{P(k-1) \phi(k-d) \varepsilon(k-1)}{\lambda+\phi^{\mathrm{T}}(k-d) P(k-1) \phi(k-d)}
$$

where

$$
P(k)=\frac{1}{\lambda}\left[P(k-1)-\frac{P(k-1) \phi(k-d) \phi^{\mathrm{T}}(k-d) P(k-1)}{\lambda+\phi^{\mathrm{T}}(k-d) P(k-1) \phi(k-d)}\right]
$$

$P(0)=P_{0}, \quad$ and $\quad \varepsilon(k-1) \triangleq \phi^{\mathrm{T}}(k-d-1) \theta(k-1)-$ $\hat{u}(k-d)$.

\subsection{Model information $\bar{B}_{f}$}

For soft-input soft-output, minimum-phase, asymptotically stable linear plants, using the first non-zero Markov parameter in $\bar{B}_{f}$ yields asymptotic convergence of $z$ to zero $[19,28]$. In this case, let $m=d$ and $\bar{H}_{d}=H_{d}$ in (23). Furthermore, if the open-loop linear plant is non-minimum-phase and the absolute values of all non-minimum-phase zeros are greater than the plant's spectral radius, then a sufficient number of Markov parameters can be used to approximate the nonminimum-phase zeros [19]. Alternatively, a phase-matching condition with $\eta>0$ is given in $[29,30]$ to construct $\bar{B}_{f}$. For MIMO Lyapunov-stable linear plants, an extension of the phase-matching-based method is given in [31]. For unstable, non-minimum-phase plants, knowledge of the locations of the non-minimum-phase zeros is needed to construct $\bar{B}_{f}$. For details, see [19, 32].

In this paper, we consider only the case where the zeros of $G_{z v}$ are either minimum-phase or on the unit circle. Therefore we set $p=1$ and let $\bar{B}_{f}=$ $\left[\begin{array}{lll}0_{1_{z} \times(d-1) l_{u}} & H_{d} & 0_{1_{z} \times(n-d) l_{u}}\end{array}\right] \in \mathbb{R}^{l_{z} \times n l_{u}}$.

\section{$4 \quad$ Numerical examples}

In this section, we present numerical examples to illustrate the response of RCAC for plants with input saturation based on constrained retrospective optimisation. The numerical examples are constructed such that the objective is to minimise the performance $z=y-w$, with $\phi(k)$ given by

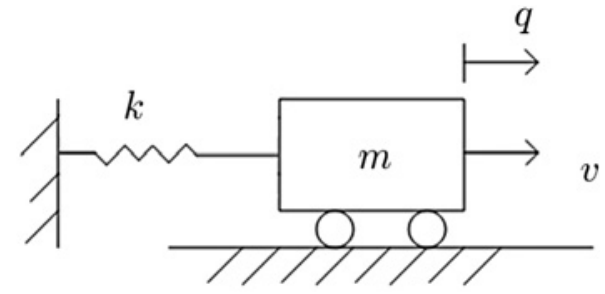

Fig. 1 Example 4.1. Mass-spring structure with single-direction force actuation

(21). In all simulations, we set $\lambda=1$ and initialise $\theta(0)$ to zero.

Example 4.1: Command following for an undamped massspring structure with single-direction force actuation. Consider the mass-spring-damper structure shown in Fig. 1 modelled by

$$
m \ddot{q}+k q=v
$$

where $m=1 \mathrm{~kg}$ and $k=30 \mathrm{~N} / \mathrm{m}$ are the mass and stiffness, $q$ and $\dot{q}$ are the position and velocity, respectively, of the mass. The saturated control $v$ is given by

$$
v=\operatorname{sat}(u)=\operatorname{sat}_{0,50}(u)
$$

The discrete-time transfer function with sampling time $T_{\mathrm{s}}=$ $0.1 \mathrm{~s}$ is given by

$$
G_{z v}(\mathbf{z})=\frac{0.004(\mathbf{z}+1)}{\mathbf{z}^{2}-1.707 \mathbf{z}+1}
$$

The goal is to bring the mass to rest at $q=0$. We consider (3) with $v=\operatorname{sat}_{0,50}(u)$. Note that this problem is related to the classical problem of controllability using positive controls considered in [33-35]. However, 33's theorem given in $[33,34]$ assumes that $B=I$, which is not the case in this example.

The adaptive controller (18) with known saturation bounds is implemented in feedback with $n_{\mathrm{c}}=5, \eta=0.0001$, $P_{0}=0.1 I$ and $\bar{B}_{f}=\left[\begin{array}{ll}0.004 & 0\end{array}\right]$.

The goal is to bring the mass to $q=0$ with singledirection force actuation. Fig. 2 shows the response with $q(0)=3 \mathrm{~m}$ and $\dot{q}(0)=5 \mathrm{~m} / \mathrm{s}$. Note that, by constraining the retrospectively optimised control $\hat{u}(k)$, the commanded control $u(k)$ is non-negative for all $k>25$.

Example 4.2: Position command following for a multi-rotor helicopter. Consider the multi-rotor helicopter illustrated in Fig. 6. The body frame $S_{\mathrm{B}}=\left\{X_{\mathrm{B}}, Y_{\mathrm{B}}, Z_{\mathrm{B}}\right\}$ is attached to the vehicle at its centre of mass $(\mathrm{CM})$ with the $Z_{\mathrm{B}}$ axis normal to the rotor plane. The reference frame $S_{\mathrm{R}}=\left\{X_{\mathrm{R}}, Y_{\mathrm{R}}, Z_{\mathrm{R}}\right\}$ is fixed on the ground at point $O$ with the $Z_{\mathrm{R}}$ axis aligned with the local vertical. The vehicle has six degrees of freedom, three of which are of rotation and the other three are of translational motion. The present example is concerned only with the translational motion, which can be described in $S_{\mathrm{R}}$ by

$$
\ddot{q}=\frac{1}{m} u+\left[\begin{array}{c}
0 \\
0 \\
-g
\end{array}\right]
$$

where $q=\left[\begin{array}{lll}q_{1} & q_{2} & q_{3}\end{array}\right]^{\mathrm{T}} \in \mathbb{R}^{3}$ describes the position of $\mathrm{CM}$, with $q_{1}$ and $q_{2}$ denoting horizontal position and $q_{3}$ representing the altitude, $u=\left[\begin{array}{lll}u_{1} & u_{2} & u_{3}\end{array}\right]^{\mathrm{T}} \in \mathbb{R}^{3}$ is the 

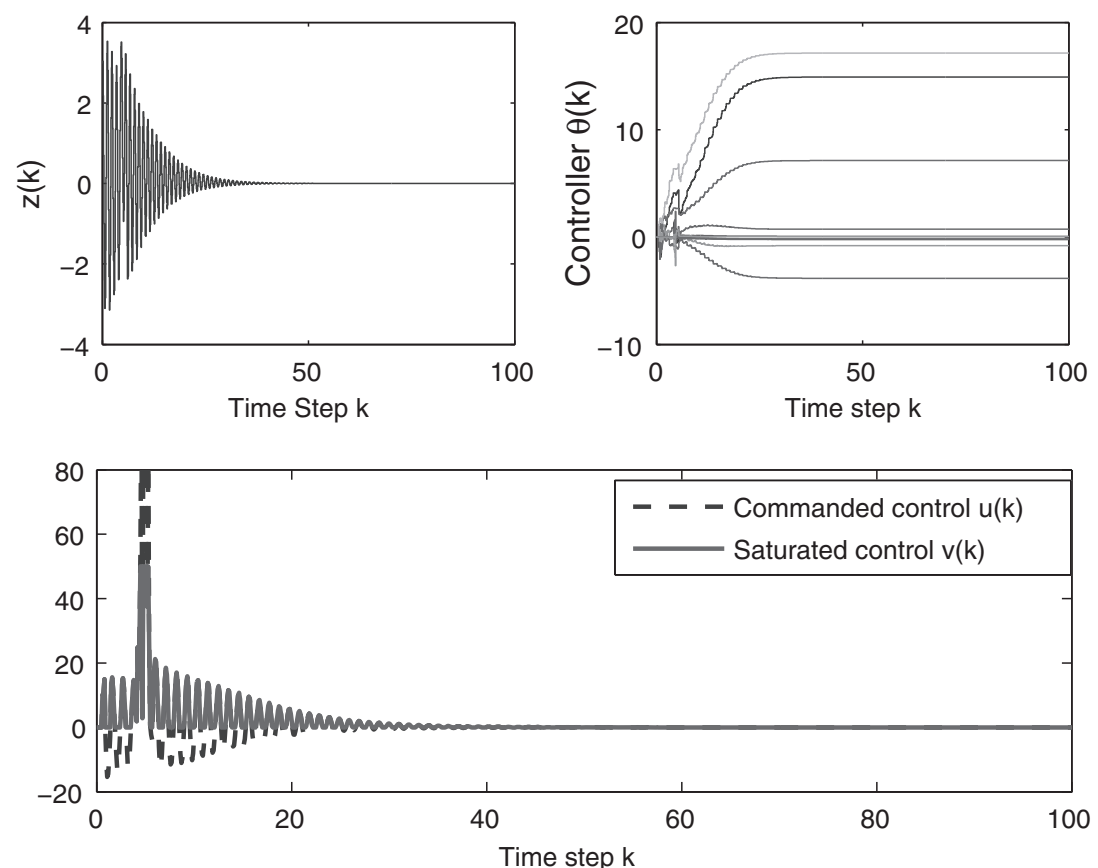

Fig. 2 Example 4.1. Command-following for an undamped mass-spring-damper structure with single-direction force actuation. The adaptive controller (18) is implemented with $n_{c}=5, \eta=0.0001, P_{0}=0.1 I$ and $\bar{B}_{f}=[0.0040]$. The goal is to bring the mass to $q=0$ with single-direction force actuation with $q(0)=3 \mathrm{~m}$ and $\dot{q}(0)=5 \mathrm{~m} / \mathrm{s}$

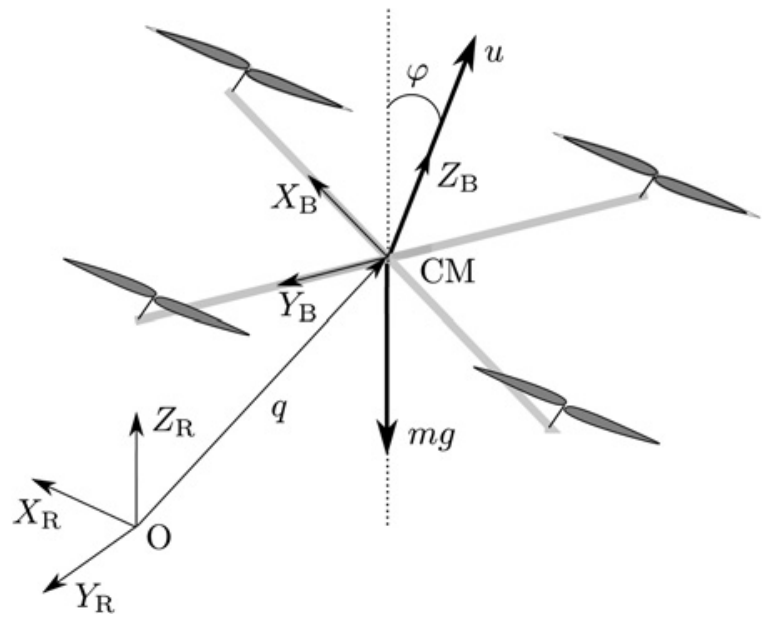

Fig. 3 Example 4.2. The multi-rotor helicopter and the body and reference frame

total thrust vector, $m=0.5 \mathrm{zg}$ is the mass of the vehicle, and $g=9.8 \mathrm{~m} / \mathrm{s}^{2}$ is the gravitational acceleration (Fig. 3). Consider the initial conditions $q(0)=\left[\begin{array}{lll}0 & 0 & 0\end{array}\right]^{\mathrm{T}}$ and $\dot{q}(0)=\left[\begin{array}{lll}0 & 0 & 0\end{array}\right]^{\mathrm{T}}$. Define the inclination angle $\varphi$ of the rotor plane to be

$$
\varphi \triangleq \cos ^{-1} \frac{u_{3}}{\|u\|}
$$

where $\|u\|$ denotes the Euclidean norm of $u$. Fig. 6 shows that $\varphi$ is the angle between the thrust vector $u$ and the $Z_{\mathrm{R}}$ axis (local vertical).

In order to implement the so-obtained controller in practice, the vehicle system is required to feature two low-level control loops: a total thrust controller and an attitude controller. In this case, the thrust magnitude $\|u\|$ serves as a command to the total thrust controller, whereas the thrust

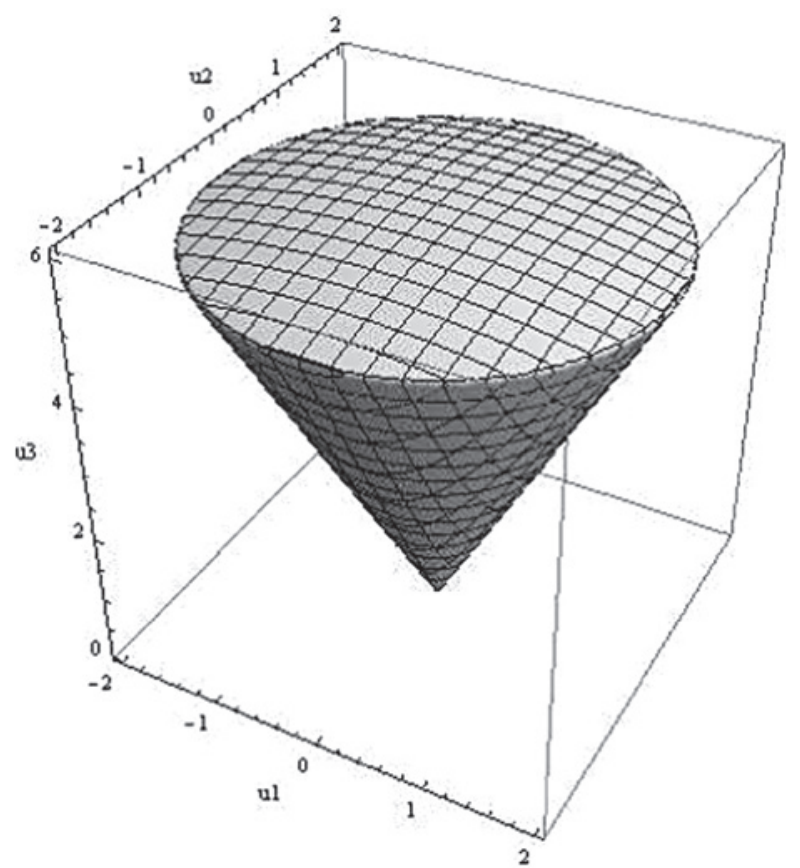

Fig. 4 Example 4.2. The convex control constraint set $\mathcal{U}$ formed by (40)-(42)

direction $u /\|u\|$ is used to generate an attitude command to the attitude controller. In this example, both low-level controllers are assumed to be accurate and significantly faster than the translational dynamics, in such a way that their command $u$ can be considered equal to its corresponding actual value.

Define the tracking error $z \in \mathbb{R}^{3}$ by

$$
z \triangleq q-w
$$



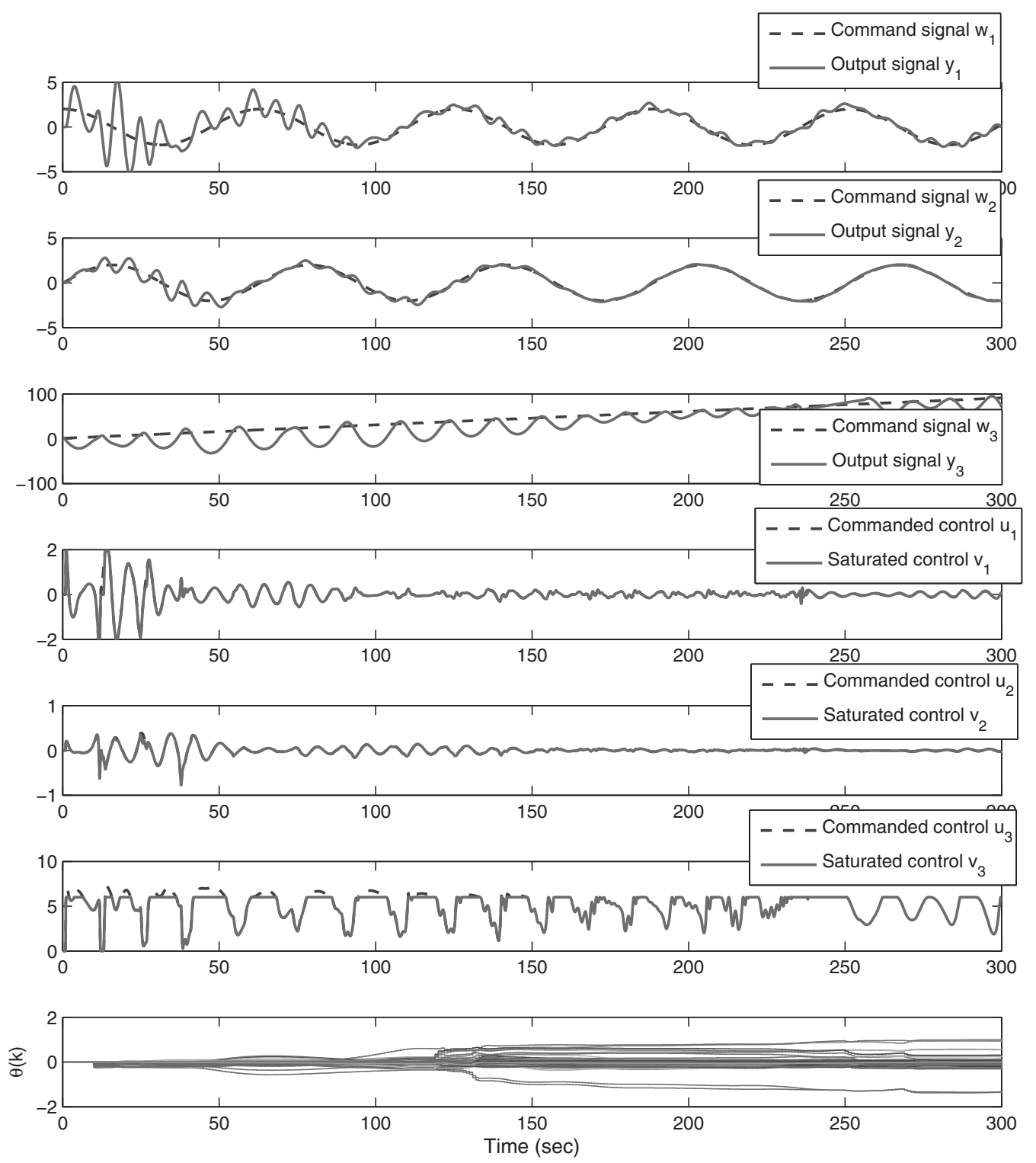

Fig. 5 Example 4.2. Command following for the multi-rotor helicopter. The adaptive controller (18) with the saturation bounds in (44) is implemented in feedback with $n_{c}=8, \eta=0, P_{0}=0.1 I, \bar{B}_{f}=\left[0.01 I_{3 \times 3} 0_{3 \times 3}\right]$ and $\theta(0)=0$. Note that $y_{1}, y_{2}, y_{3}$ follow the commands $w_{1}, w_{2}$ and $w_{3}$ after the transient

where $w=\left[\begin{array}{lll}w_{1} & w_{2} & w_{3}\end{array}\right] \in \mathbb{R}^{3}$ denotes a position command. Consider

$$
w(t)=\left[\begin{array}{c}
2 \cos (0.1 t) \\
2 \sin (0.1 t) \\
0.3 t+1
\end{array}\right]
$$

Let $0 \leq \varphi_{\max } \leq 90^{\circ}$ and $u_{\max }>0$ denote the maximum allowable values of $\varphi$ and $\|u\|$, respectively. Consider $\varphi_{\max }=20^{\circ}$ and $u_{\max }=6 \mathrm{~N}$.

The control problem is to construct a feedback law for $u$ that minimises $\|z\|$ subject to

$$
\begin{aligned}
\frac{\sqrt{u_{1}^{2}+u_{2}^{2}}}{\|u\|} & \leq \sin \varphi_{\max } \\
u_{3} & \geq 0
\end{aligned}
$$

and

$$
\|u\| \leq u_{\max }
$$

The inequalities (40)-(42) form the conic convex control constraint set $\mathcal{U}$ illustrated in Fig. 4. The problem of minimising the retrospective cost function on $\mathcal{U}$ can thus be rewritten as the following second-order cone programming (SOCP) problem

$$
\min J(\hat{U}(k), k)
$$

subject to

$$
\|P \hat{U}(k)\|_{2} \leq Q \hat{U}(k) \quad \text { and } \quad\|\hat{U}(k)\|_{2} \leq 6
$$

where

$$
P \triangleq\left[\begin{array}{ccccc}
1 & 0 & 00 & 1 & 0 \\
0 & 0 & 0 & &
\end{array}\right]
$$

and

$$
Q \triangleq \tan \left(\varphi_{\max }\right)\left[\begin{array}{lll}
0 & 0 & 1
\end{array}\right]^{\mathrm{T}}
$$

The non-linear programming method SOCP available in the CVX toolbox [23] is used to solve the above optimisation problem. 

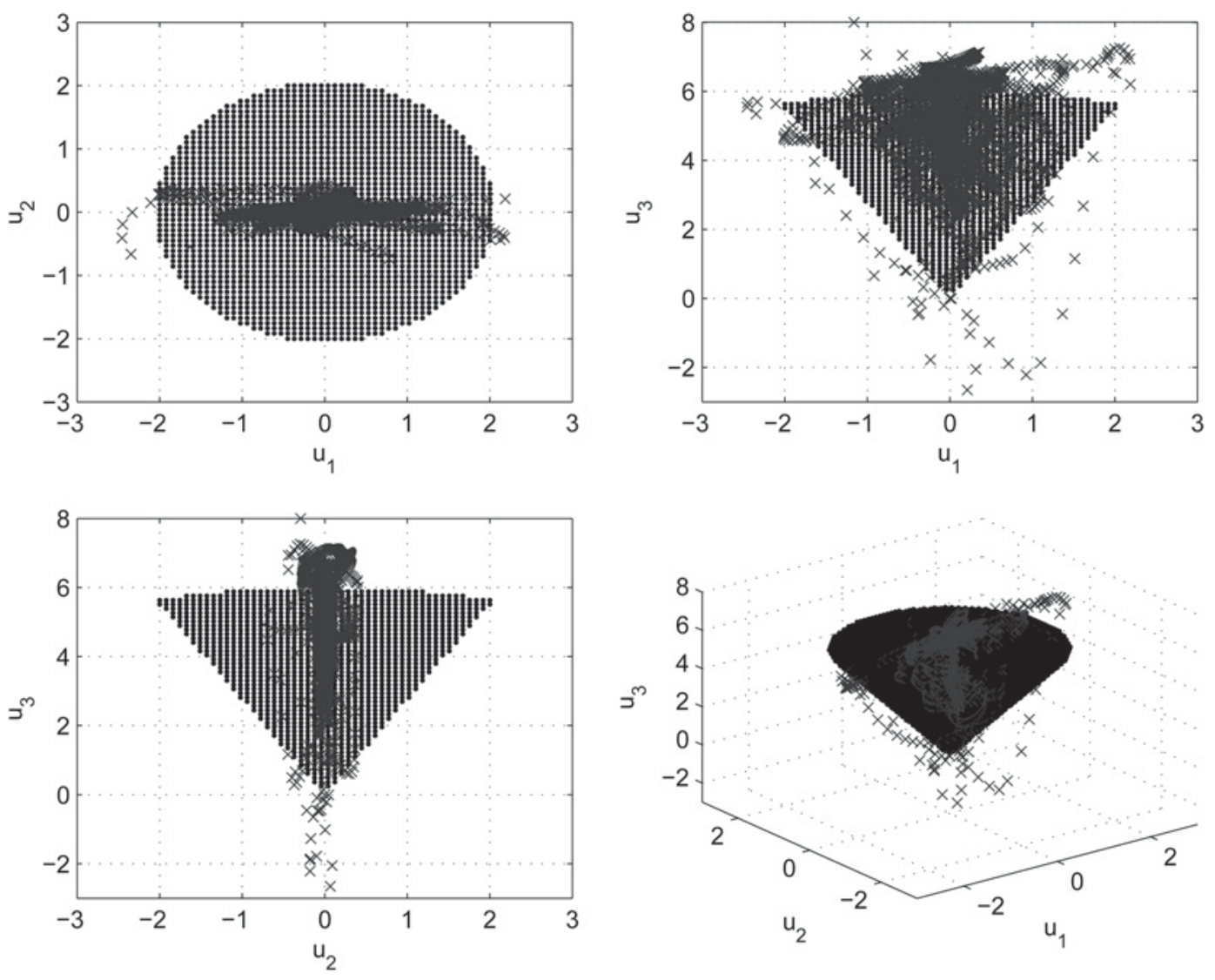

Fig. 6 Example 4.2. The adaptive controller (18) with known saturation bounds in (44) is implemented in feedback with $n_{c}=8$, $\eta=0$, $P_{0}=0.1 I, \bar{B}_{f}=\left[\begin{array}{lll}0.01 I_{3 \times 3} & 0_{3 \times 3}\end{array}\right]$ and $\theta(0)=0$. The black dots represent the constraint set in (40) and (42), and the blue dots represent the unsaturated controller output $u$. The blue crosses outside the boundary of the constraint (black dots region) are because of the transient behaviour of RLS update in (31) and (32)

Next, a state space representation of the multi-rotor helicopter is given by

$$
\begin{aligned}
{\left[\begin{array}{c}
\dot{q} \\
\ddot{q}
\end{array}\right] } & =\left[\begin{array}{ll}
0_{3 \times 3} & I_{3 \times 3} \\
0_{3 \times 3} & 0_{3 \times 3}
\end{array}\right]\left[\begin{array}{c}
q \\
\dot{q}
\end{array}\right]+\left[\begin{array}{c}
0_{3 \times 3} \\
\frac{1}{m} I_{3 \times 3}
\end{array}\right] v+\left[\begin{array}{c}
d_{1} \\
d_{2} \\
0 \\
0 \\
0 \\
-g
\end{array}\right] \\
y & =\left[\begin{array}{ll}
I_{3 \times 3} & 0_{3 \times 3}
\end{array}\right]\left[\begin{array}{c}
q \\
\dot{q}
\end{array}\right] \\
z & =y-w
\end{aligned}
$$

where the horizonal wind velocity $d_{1}=d_{2}=0.1 \mathrm{~m} / \mathrm{s}$ and $v=\operatorname{Sat}(u) \in \mathcal{U}$ is the saturated control input given by

$$
v=\left[\begin{array}{c}
v_{1} \\
v_{2} \\
v_{3}
\end{array}\right]=\operatorname{Sat}(u)=\left[\begin{array}{c}
\operatorname{Sat}_{1}\left(u_{1}, u_{2}, u_{3}\right) \\
\operatorname{Sat}_{2}\left(u_{1}, u_{2}, u_{3}\right) \\
\operatorname{Sat}_{3}\left(u_{3}\right)
\end{array}\right]
$$

where (see (51) and (52))

$$
\operatorname{Sat}_{3}\left(u_{3}\right) \triangleq \operatorname{sat}_{0,6}\left(u_{3}\right)
$$

and

$$
\vartheta \triangleq \operatorname{atan} 2\left(u_{2}, u_{1}\right)=2 \arctan \frac{u_{2}}{\sqrt{u_{1}^{2}+u_{2}^{2}}+u_{1}}
$$

Note that the function Sat in (50) satisfies 3.1. Next, we discretise (47)-(49) using zero-order-hold with sampling time $T_{\mathrm{s}}=0.01 \mathrm{~s}$. The adaptive controller (18) with knowledge of the saturation (44) is implemented in feedback with $n_{\mathrm{c}}=8, \eta=0, P_{0}=0.1 \mathrm{I}, d=1, H_{1}=I_{3 \times 3}$ and we let $\bar{B}_{f}=\left[0.01 I_{3 \times 3} \quad 0_{3 \times 3}\right]$.

Fig. 5 shows the time history of $y_{1}, y_{2}$ and $y_{3}$ of the helicopter. The transient especially along $y_{3}$ direction is owing to the fact that (47)-(49) is unstable, the discretised equivalent of (47)-(49) has non-minimum-phase zeros at -1 , and the horizontal wind velocity $d_{1}, d_{2}$ and the gravitational acceleration $g$ are unmodelled. Furthermore, we initialise the adaptive controller at $\theta(0)=0$. Note that the commanded control signal $u$ does not exhibit integrator windup

$$
\begin{aligned}
& \operatorname{Sat}_{1}\left(u_{1}, u_{2}, u_{3}\right) \triangleq \begin{cases}u_{1} & \text { if } u_{1} \leq \operatorname{Sat}_{3}\left(u_{3}\right) \tan \varphi_{\max } \cos \vartheta \\
\operatorname{Sat}_{3}\left(u_{3}\right) \tan \varphi_{\max } \cos \vartheta & \text { otherwise }\end{cases} \\
& \operatorname{Sat}_{2}\left(u_{1}, u_{2}, u_{3}\right) \triangleq \begin{cases}u_{2} & \text { if } u_{2} \leq \operatorname{sat}_{3}\left(u_{3}\right) \tan \varphi_{\max } \sin \vartheta \\
\operatorname{Sat}_{3}\left(u_{3}\right) \tan \varphi_{\max } \sin \vartheta & \text { otherwise }\end{cases}
\end{aligned}
$$




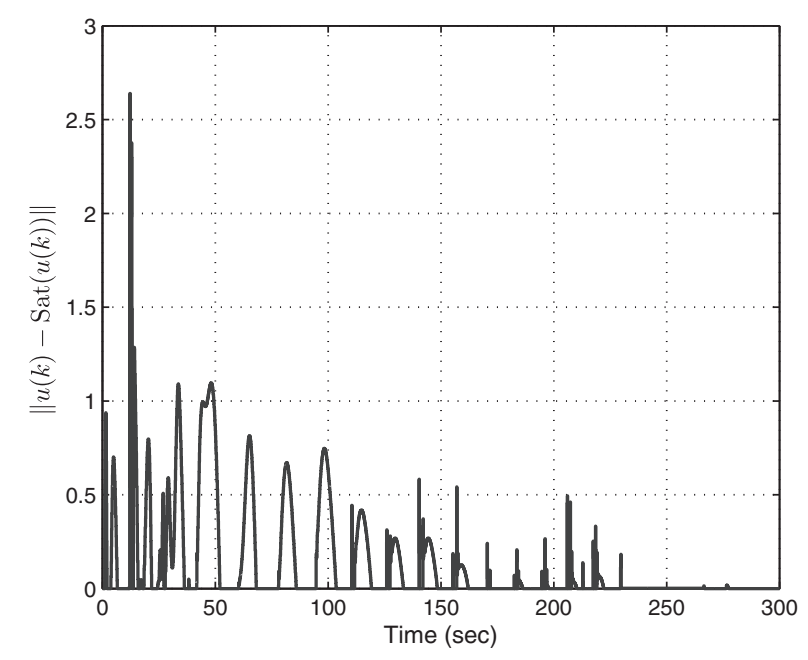

Fig.7 Example 4.2. At each time step, the distance between the commanded control $u(k)$ (blue crosses that are outside the control constraint set $\mathcal{U}$ in Fig. 6 and the saturated control sat $(u(k))$

and remains bounded as shown in Fig. 6, where the black dots represent the control constraint set $\mathcal{U}$, and the blue crosses represent the commanded control $u$. Note that the blue crosses outside the control constraint set $\mathcal{U}$ (black dots region) are caused by the transient behaviour of RLS update in (31) and (32). Fig. 7 shows that at each time step, the distance between the unsaturated commanded control $u(k)$ (blue crosses that are outside the control constraint set $\mathcal{U}$ in Fig. 6 and the saturated control $v(k)$.

\section{Conclusions}

Adaptive control based on constrained retrospective cost optimisation was applied to command following for Hammerstein systems with multi-variable convex input saturation. We numerically demonstrated that convex optimisation applied to the retrospective cost can improve the tracking performance when following commands in the presence of saturation. We also applied this technique to a multi-rotor helicopter command-following problem by formulating the multi-input constrained retrospective cost function as a second-order cone optimisation problem. With this approach, RCAC was shown to adapt to these constraints. The numerical results motivate future research on the stability analysis of RCAC under input saturation.

\section{Acknowledgment}

We wish to thank Jesse Hoagg and Alexey Morozov for helpful discussions.

\section{References}

1 Bernstein, D.S., Michel, A.N.: 'A chronological bibliography on saturating actuators,' Int. J. Robust Nonlinear Control, 1995, 5, pp. $375-380$

2 Zaccarian, L., Teel, A.R.: 'Modern anti-windup synthesis: control augmentation for actuator saturation' (Princeton University Press, 2011)

3 Hippe, P.: 'Windup in control: its effects and their prevention' (Springer, 2006)
4 Hanus, R., Kinnaert, M., Henrotte, J.L.: 'Conditioning technique, a general anti-windup and bumpless transfer method,' Automatica, 1987 , 23, (6), pp. 729-739

5 Åström, K.J., Rundqwist, L.: 'Integrator windup and how to avoid it,' Proc. American Control Conf., Pittsburgh, PA, June 1989, pp. 1693-1698

6 Zheng, A., Kothare, M.V., Morari, M.: 'Anti-windup design for internal model control,' Int. J. Control, 1993, 60, pp. 1015-1024

7 Tarbouriech, S., Turner, M.: 'Anti-windup design: an overview of some recent advances and open problems,' IET Control Theory Appl., 2009, 3, (1), pp. 1-19

$8 \mathrm{Hu}, \mathrm{T}$., Lin, Z.: 'Control systems with actuator saturation: analysis and design' (Birkhäuser, 2001)

9 Glattfelder, A., Schaufelberger, W.: 'Control systems with input and output constraints' (Springer, 2003)

10 Kapila, T., Grigoriadis, K.: 'Actuator saturation control' (CRC Press, 2002)

11 Tarbouriech, S., Garcia, G., daSilvaJr., J.M.G., Queinnec, I.: 'Stability and stabilization of linear systems with saturating actuators' (Springer, 2011)

12 Zaccarian, L., Teel, A.R.: 'Modern anti-windup synthesis: control augmentation for actuator saturation' (Princeton University Press, 2011)

13 Annaswamy, A.M., Wong, J.-E.: 'Adaptive control in the presence of saturation non-linearity,' Int. J. Adaptive Control Sig. Proc., 1998, 11, pp. 3-19

14 Teo, J., How, J.P.: 'Anti-windup compensation for nonlinear systems via gradient projection: Application to adaptive control,' Proc. IEEE Conf. on Dec. Control, Shanghai, China, December 2009, pp. 6910-6916

15 Coffer, B.J., Hoagg, J.B., Bernstein, D.S.: 'Cumulative retrospective cost adaptive control of systems with amplitude and rate saturation,' Proc. American Control Conf., San Francisco, CA, June 2011, pp. 2344-2349

16 Do, H.M., Başar, T., Choi, J.Y.: 'An anti-windup design for single input adaptive control systems in strict feedback form,' Proc. American Control Conf., Boston, MA, June 2004, pp. 2551 - 2556

17 Tyan, F., Bernstein, D.S.: 'Global stabilization of systems containing a double integrator using a saturated linear controller,' Int. J. Robust Nonlinear Control, 1999, 9, (15), pp. 1143-1156

18 Hoagg, J.B., Santillo, M.A., Bernstein, D.S.: 'Discrete-time adaptive command following and disturbance rejection for minimum phase systems with unknown exogenous dynamics,' IEEE Trans. Autom. Control, 2008, 53, pp. 912-928

19 Santillo, M.A., Bernstein, D.S.: 'Adaptive control based on retrospective cost optimization,' AIAA J. Guid. Control Dyn., 2010, 33, pp. 289-304

20 Hoagg, J.B., Bernstein, D.S.: 'Retrospective cost model reference adaptive control for nonminimum-phase systems,' AIAA J. Guid. Control Dyn., 2012, 35, pp. 1767-1786

21 Hoagg, J.B., Bernstein, D.S.:, 'Retrospective cost adaptive control for nonminimum-phase discrete-time systems part 1: The ideal controller and error system; part 2: The adaptive controller and stability analysis,' Proc. IEEE Conf. Dec. Control, Atlanta, GA, December 2010, pp. 893-904

22 D'Amato, A.M., Bernstein, D.S.: 'Adaptive forward-propagating input reconstruction for nonminimum-phase systems,' Proc. American Control Conf., Montreal, Canada, June 2012, pp. 598-603

23 Grant, M., Boyd, S.: (2013, March) CVX: Matlab software for disciplined convex programming, version 2.0 . http://cvxr.com/cvx/

24 Morozov, A., D'Amato, A.M., Hoagg, J.B., Bernstein, D.S.: 'Retrospective cost adaptive control for nonminimum-phase systems with uncertain nonminimum-phase zeros using convex optimization,' Proc. American Control Conf., San Francisco, CA, June 2011, pp. $1188-2293$

25 Yan, J., Bernstein, D.S.: 'Minimum modeling retrospective cost adaptive control of uncertain Hammerstein systems using auxiliary nonlinearities,' Int. J. Control, 2014, 87, pp. 483-505

26 Åström, K.J., Wittenmark, B.: 'Adaptive control' (Addison-Wesley, 1995)

27 Goodwin, G.C., Sin, K.S.: 'Adaptive filtering, prediction and control (Prentice Hall, 1984)

28 D'Amato, A.M., Sumer, E.D., Bernstein, D.S.: 'Frequency-domain stability analysis of retrospective-cost adaptive control for systems with unknown nonminimum-phase zeros,' Proc. IEEE Conf. on Dec. Control, Orlando, FL, December 2011, pp. 1098-1103

29 Sumer, E.D., D’Amato, A.M., Morozov, A.M., Hoagg, J.B., Bernstein, D.S.: 'Robustness of retrospective cost adaptive control to 


\section{www.ietdl.org}

Markov-parameter uncertainty,' Proc. IEEE Conf. on Dec. Control, Orlando, FL, December 2011, pp. 6085-6090

30 Sumer, E.D., Holzel, M.H., D’Amato, A.M., Bernstein, D.S.: 'FIR-based phase matching for robust retrospective-cost adaptive control,' Proc. American Control Conf., Montreal, Canada, June 2012, pp. 2707-2712

31 Sumer, E.D., Bernstein, D.S.: 'Retrospective cost adaptive control with error-dependent regularization for mimo systems with unknown nonminimum-phase transmission zeros,' Proc. AIAA Guid. Nav. Control Conf., Minneapolis, MN, August 2012, AIAA-2012-4070
32 Hoagg, J.B., Bernstein, D.S.: 'Cumulative retrospective cost adaptive control with RLS-based optimization,' Proc. American Control Conf., Baltimore, MD, June 2010, pp. 4016-4021

33 Brammer, R.F.: 'Controllability in linear autonomous systems with positive controllers,' SIAM J. Control, 1972, 10, pp. 339-353

34 Jacobson, D.H., Margin, D.H., Pachter, M., Geveci, T.: 'Extensions of linear-quadratic control theory' (Springer, 1980)

35 Leyva, H., Solís-Daun, J., Suárez, R.: 'Global CLF stabilization of systems with control inputs constrained to a hyperbox,' SIAM J. Control Opt., 2013, 51, pp. 745-766 